\title{
Mechanisms by which CXCR4/CXCL12 cause metastatic behavior in pancreatic cancer
}

\author{
JIANBO ZHANG ${ }^{1}$, CHENGXIN LIU $^{2}$, XINKAI MO ${ }^{1}$, HUAN SHI $^{2}$ and SHENG LI $^{3}$ \\ Departments of ${ }^{1}$ Pathology, ${ }^{2}$ Oncology and ${ }^{3}$ Hepatology, Shandong Cancer Hospital Affiliated to Shandong \\ University, Shandong Academy of Medical Sciences, Jinan, Shandong 250117, P.R. China
}

Received May 2, 2016; Accepted January 17, 2017

DOI: $10.3892 / \mathrm{ol} .2017 .7512$

\begin{abstract}
C-X-C motif chemokine receptor (CXCR) 4/CXCL12 is associated with tumor invasion and metastasis in pancreatic cancer. The present study aimed to investigate the possible mechanisms behind this process by studying the association between the expression of CXCR4 and numerous molecular markers. A total of 60 patients with pancreatic cancer who had been treated with radical surgery between July 2012 and February 2016 were included in the present study. The expression of CXCR4/CXCL12 in primary pancreatic cancer lesions, tissues adjacent to cancerous tissue, non-cancerous pancreatic tissues and in the surrounding lymph nodes was evaluated by immunohistochemistry. Expression levels of four candidate biomarkers [vascular endothelial growth factor-C (VEGF-C), Ki-67, matrix metalloproteinase 2 (MMP-2) and $\beta$-catenin] were also evaluated. The correlation between CXCR4 and these four biomarkers was assessed.CXCR4 (CXCL12) expression levels were higher in pancreatic cancer $56.7 \%$ (86.7\%), paracancerous tissue $50.0 \%(85.0 \%)$ and surrounding lymph nodes $53.3 \%$ (80.0\%), compared with in normal tissues $18.3 \%(45.0 \%)$. CXCR4 expression was significantly associated with the lymph node metastasis of tumors $(\mathrm{P}=0.001)$, pathological type $(\mathrm{P}=0.037)$ and tumor-node-metastasis stage $(\mathrm{P}=0.031)$. CXCR4 expression exhibited a positive correlation with VEGF-C ( $\mathrm{r}=0.417$; $\mathrm{P}=0.001)$, Ki-67 ( $\mathrm{r}=0.316 ; \mathrm{P}=0.014), \mathrm{MMP}-2(\mathrm{r}=0.284$; $\mathrm{P}=0.028)$ and $\beta$-catenin $(\mathrm{r}=0.368 ; \mathrm{P}=0.04)$. Furthermore, logistic regression analysis revealed VEGF-C $(\beta=1.722$; $\mathrm{P}=0.005)$ and $\mathrm{Ki}-67(\beta=1.196 ; \mathrm{P}=0.047)$ to be two biomarkers that cause metastasis via CXCR4. CXCR4/CXCL12 is closely associated with tumor grade and lymphatic metastasis. VEGF-C and Ki-67 are two important biomarkers, through
\end{abstract}

Correspondence to: Professor Sheng Li, Department of Hepatology, Shandong Cancer Hospital Affiliated to Shandong University, Shandong Academy of Medical Sciences, 440 Jiyan Road, Jinan, Shandong 250117, P.R. China

E-mail: 1wtdzyyx@163.com

Key words: CXCR4/CXCL12, pancreatic cancer, mechanism, biomarkers which CXCR4 initiates metastatic behavior in pancreatic cancer. Therefore, angiogenesis inhibitors will continue to be effective agents in treating pancreatic cancer.

\section{Introduction}

Pancreatic cancer is a type of digestive malignant tumor, and has a poor prognosis (1). Patients with pancreatic cancer often experience few symptoms in the early stages of the disease, and local invasion and distant metastases typically occur quickly (2). Despite aggressive surgical resection and advances in systemic treatment methods, the mortality rate of pancreatic cancer ranks fourth worldwide, with a five-year survival rate of $<5 \%$ and a continuously increasing rate of mortality and morbidity $(3,4)$. As a result, the investigation and understanding of the mechanisms underlying the invasion and metastasis of pancreatic cancer is important in the diagnosis and treatment of this disease.

In a number of previous studies, the interaction of C-X-C motif chemokine receptor (CXCR) 4 with CXCL12 has been observed to perform an important role in tumor proliferation, invasion, angiogenesis, metastasis and migration in numerous types of cancer (5-7). It is also considered that the CXCR4/CXCL12 axis is significantly associated with the prognosis of patients with pancreatic tumors, and may become a novel target for the treatment of tumors (8). In a previous study, the regulation of angiogenesis and lymphangiogenesis was demonstrated to be a possible mechanism by which CXCR4 influenced the progression of pancreatic cancer using quantitative-polymerase chain reaction (9). However, this study involved only 30 patients and no critical biomarker was identified.

Therefore, the aim of the present study was to test the association between the expression of CXCR4 and clinicopathological factors in a larger sample size. The present study focused on the association between CXCR4 and vascular endothelial growth factor-C (VEGF-C), and the effect of CXCR4 on angiogenesis in pancreatic cancer. Immunohistochemistry was used to study the association among biomarkers [(CXCR4, VEGF-C, $\beta$-catenin, Ki-67 and matrix metalloproteinase 2 (MMP-2)] in order to investigate the relevant signaling pathways and corresponding proteins that are involved in the complex molecular network underlying the invasion and metastasis of pancreatic cancer. 


\section{Materials and methods}

Sample collection. Tissue samples were obtained from 60 patients who were diagnosed with and treated for pancreatic cancer by pathological section analysis at the Shandong Tumor Hospital (Jinan, China) between July 2012 and February 2016. The clinical information for all patients was complete. Informed consent was obtained from each patient. Patients consisted of 32 males and 28 females, with an average age of 57.5 years (range, 34-74). Samples of the pancreatic tumor, paracancerous tissues (obtained $<2 \mathrm{~cm}$ from the tumor), normal pancreas and the lymph nodes surrounding the pancreas were obtained through macroscopic curative resection. Among the 60 samples, 41 were derived from the head of the pancreas and the remaining 19 originated from alternate areas. A total of 54 tissue samples were defined as being ductal adenocarcinoma, and 6 were other pathological types. In addition, 27 of the samples were poorly differentiated and 33 cases were well/moderately differentiated.

There were 23 patients classified as having stage I-II pancreatic cancer and 37 patients classified as having stage III-IV pancreatic cancer [according to the 7th edition of tumor-node-metastasis (TNM) staging system provided by the American Joint Committee on Cancer] (10). In total, 35 patients were identified as having lymph nodes metastasis whilst the remaining 25 cases did not exhibit lymph nodes metastasis. The present study included 42 cases for which the maximum lesion diameter was $>3 \mathrm{~cm}$, whilst the maximum diameters for the other 18 cases were $\leq 3 \mathrm{~cm}$. All tissues were fixed in $4 \%$ neutral buffered formalin for $24 \mathrm{~h}$ at $37^{\circ} \mathrm{C}$, embedded in paraffin and sectioned into $4 \mu \mathrm{m}$ slices. New sections were then stained with hematoxylin and eosin to verify diagnosis and perform immunohistochemical analysis.

Immunohistochemical assays. The sections were manipulated to immunohistochemical staining using the Dako Envision System method (Dako; Agilent Technologies, Inc., Santa Clara, CA, USA), according to the manufacturer' protocol. A positive section of company configuration (Dako; Agilent Technologies, Inc.) was used as a positive control and PBS was employed as the negative control. The sections were heated for $1 \mathrm{~h}$ at $65^{\circ} \mathrm{C}$ and dewaxing was performed subsequently. Sections were then washed with a graded ethanol series $(95$, 85 and $75 \%$ ), incubated with ethanol containing $3 \% \mathrm{H}_{2} \mathrm{O}_{2}$ for $10 \mathrm{~min}$ to block endogenous peroxidase and microwaved at $98^{\circ} \mathrm{C}$ for $15 \mathrm{~min}$ to retrieve the antigen. Subsequent to cooling for $10 \mathrm{~min}$ at room temperature, sections were rinsed with PBS and incubated overnight at $4^{\circ} \mathrm{C}$ with the following primary antibodies: CXCR4 (dilution, 1:40; cat no. 374606; mouse monoclonal; R\&D Systems, Inc., Minneapolis, MN, USA), CXCL12 (dilution, 1:100; cat no. 79018.111; mouse monoclonal; R\&D Systems, Inc.), cluster of differentiation 34 (dilution, 1:80; cat no. QBEnd/10; mouse monoclonal; Fuzhou Maixin Biotech. Co., Ltd., Fuzhou, China), VEGF-C (dilution, 1:200; cat no. VG1; mouse monoclonal; Fujian Gutian Yuanhang Medical Co., , Ltd.), Ki-67 (dilution, 1:200; cat no. K-2; mouse monoclonal; ZSGB-BIO Co., Ltd., Beijing, China), $\beta$-catenin (dilution, 1:100; cat no. CAT-5H10; mouse monoclonal; ZSGB-BIO) and MMP-2 (dilution, 1:200; cat no. ab37150; rabbit polyclonal; Abcam, Cambridge, UK). Sections were subsequently incubated with a goat anti-mouse horseradish peroxidase conjugated-immunoglobulin $\mathrm{G}$ antibody (dilution, 1:500; cat no. 111-035-003; Thermo Fisher Scientific, Inc., Waltham, MA, USA) multimer for $15 \mathrm{~min}$ at $37^{\circ} \mathrm{C}$. Subsequent to rinsing with PBS, the sections were visualized using 3,3'diaminobenzidine, and the slides were counterstained with hematoxylin.

Interpretation of result. The double blind reading method was utilized in the present study. Cells with a cytomembrane and cytoplasm that was stained by the CXCL12/CXCR4 antibody were defined as CXCL12/CXCR4 positive. Similarly its corresponding antibody stained the cytoplasm of VEGF-C positive cells. Ki-67 positive cells expressed nuclear staining, MMP-2 positive cells exhibited cytoplasmic staining and $\beta$-catenin positive cells demonstrated cytomembrane or cytoplasmic staining.

Each section should count $\geq 10400$-fold fields of view, and cells exhibiting brown granules were considered to be positive cells (positive for the targeted biomarkers). Slices were graded based on the percentage of stained cells and the staining strength in the semi-quantitative integral method detailed below. The percentage of stained cells was classified as follows: $\leq 10 \%, 0$ points; $>10 \%$ and $<25 \%, 1$ point; $\geq 25$ and $\leq 50 \%, 2$ points; $>50 \%, 3$ points. A staining percentage $>10 \%$ was classified as being a positive range. Staining intensity was dependent on the shade of the color: Without staining, 0 points; pale yellow, 1 point; brown, 2 points; dark brown, 3 points. The final score demonstrated the positive level of the slice and was determined by the product of the two scores above: A staining score $\leq 1$ was considered negative (-), 2-3 points indicated positive staining $(+)$ and $\geq 4$ points demonstrated strong positive staining (++; Fig. 1).

Statistical analysis. Statistical analysis was conducted using SPSS 19.0 (IBM SPSS, Armonk, NY, USA). The $\chi^{2}$-test was used for univariate analysis to identify the clinicopathological factors that may affect CXCR4, including gender, age, location, differentiation, pathological type, tumor size, lymph node metastasis and TNM stage. Logistic regression and Spearman's rank test were used for multivariate analysis of CXCR4 with these four biomarkers. $\mathrm{P}<0.05$ was considered to indicate a statistically significant difference.

\section{Results}

Expression of CXCR4 in pancreatic tumors, paracancerous tissues, normal pancreatic tissue and in the lymph nodes surrounding the pancreas. The level of CXCR4 protein expression is low in normal pancreatic tissue, and high in pancreatic tumor tissues, paracancerous tissues and the lymph nodes surrounding the pancreas, with rates of positive expression of CXCR4 (CXCL12) being 18.3\% (45.0\%), 56.7\% (86.7\%), 50.0\% (85.0\%) and 53.3\% (80.0\%), respectively. No significant differences were identified between pancreatic tumor tissues, paracancerous tissues and lymph nodes $(\mathrm{P}>0.05)$; however, all three tissue types were significantly different compared with the normal pancreatic tissue sample $(\mathrm{P}<0.05$; Table I). 

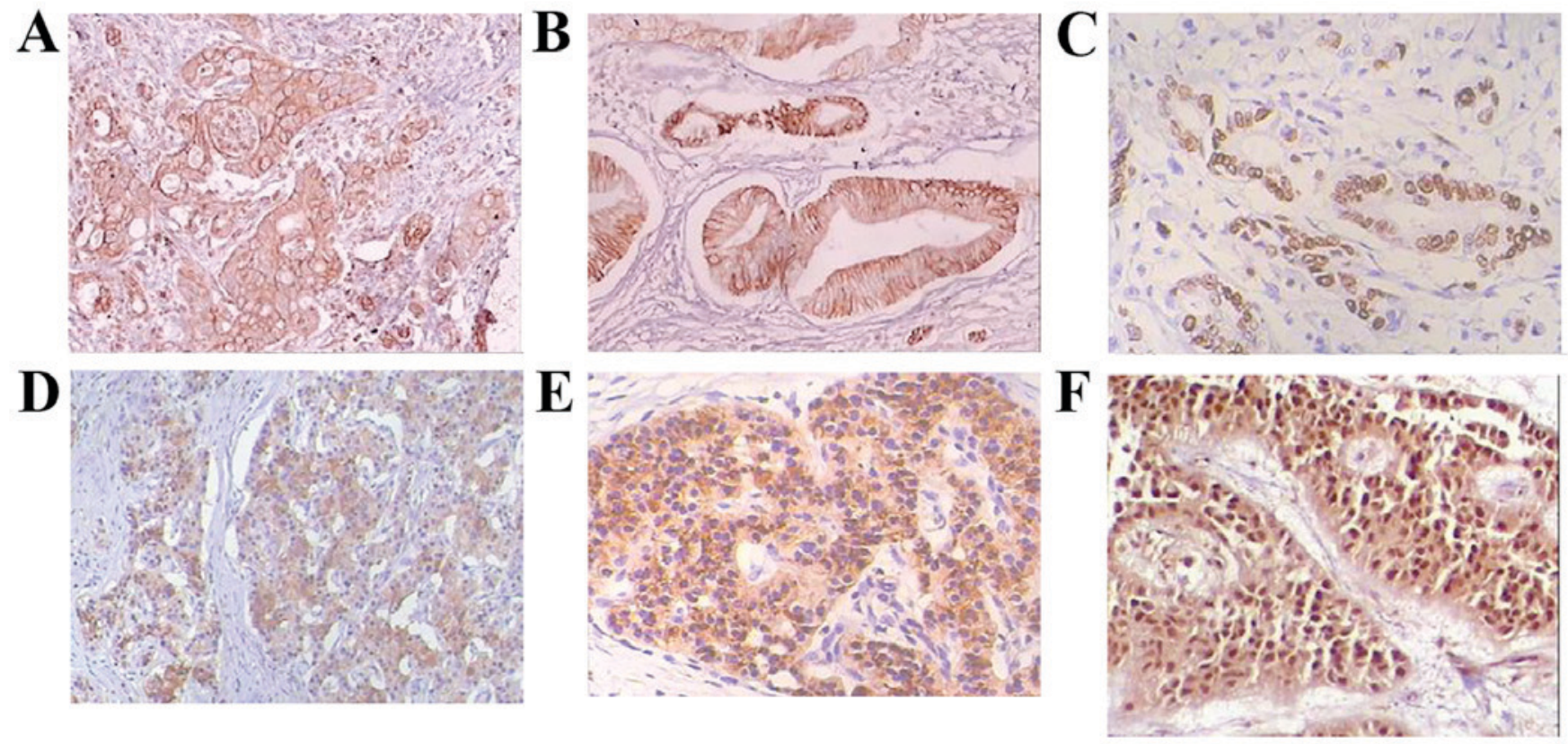

Figure 1. Immunohistochemical staining of pancreatic cancer tissue using hematoxylin and eosin staining. (A) CXCR4 positive expression, magnification x100; (B) CXCL12 positive expression, magnification x100; (C) Ki-67 positive expression, magnification x200; (D) MMP-2 positive expression, magnification $\mathrm{x} 100$; (E) $\beta$-catenin positive expression, magnification $\mathrm{x} 200$; (F) VEGF-C positive expression, magnification $\mathrm{x} 200$. CXCR, C-X-C motif chemokine receptor; CXCR, C-X-C motif chemokine ligand; MMP, matrix metalloproteinase; VEGF, vascular endothelial growth factor.

The correlation between clinicopathological factors and CXCR4 expression. In order to illuminate the role of CXCR4 in the progression of pancreatic cancer, the present study also analyzed the association between the expression profiles of CXCR4 and the grade and tumor stage in 60 patients. CXCR4 expression was significantly associated with tumor lymph node metastasis $(\mathrm{P}=0.001)$, pathological type $(\mathrm{P}=0.037)$ and TNM stage $(\mathrm{P}=0.031)$. However, no correlation was observed between CXCR4 expression and patient age, gender, tumor location, tumor differentiation or tumor size (Table II).

Correlation analysis between CXCR4 and biomarkers. In order to analyze the correlation of CXCR4 with VEGF-C, Ki-67, MMP-2 and $\beta$-catenin, the present study detected their expression levels and performed data analysis using the Spearman's rank test. The results demonstrate that CXCR4 exhibited a positive correlation with VEGF-C ( $\mathrm{r}=0.417$; $\mathrm{P}=0.001)$, Ki-67 ( $\mathrm{r}=0.316 ; \mathrm{P}=0.014), \mathrm{MMP}-2$ ( $\mathrm{r}=0.284$; $\mathrm{P}=0.028)$ and $\beta$-catenin $(\mathrm{r}=0.369 ; \mathrm{P}=0.004$; Table III). Furthermore, logistic regression analysis revealed VEGF-C $(\beta=1.722 ; \mathrm{P}=0.005)$ and $\mathrm{Ki}-67(\beta=1.196 ; \mathrm{P}=0.047)$ to be the independent factors (Table IV).

\section{Discussion}

The morbidity of pancreatic cancer is $\sim 10 / 100,000$ and the rate of incidence is continuously increasing worldwide (11). Pancreatic cancer accounted for $4-5 \%$ of cancer-associated mortality in 2015 worldwide and, as a result of a high frequency of local or distant metastasis, its prognosis is poor $(3,11-14)$. Tumor migration is controlled by its chemokine receptors and the corresponding chemokine expressed in target organ. When the chemokine combines with its specific receptor, it may provoke the aggregation of actin and induce the movement and migration of tumor cells.

The CXCL12/CXCR4 axis is the typical representation used to describe the tendency of tumor cells to metastasize; the expression of CXCL12 and CXCR4 has been observed to increase in breast cancer, glioma and melanoma (15-18). As suggested by the present study, the mechanism by which a pancreatic tumor cell may metastasize to a specify organ could be as follows: Firstly, tumor cells proliferate in primary lesions, and there is an overexpression of CXCR4 on the surface of cells. Secondly, CXCL12, which is expressed on the surface of the target organ, may promote tumor cells separate from tumor tissue and activate several adhesion molecules of cells and induce them to secrete more MMP and VEGF in order to dissolve the extracellular matrix (ECM). Subsequently, tumor cells may cross the lymph vessel and blood vessel walls of the tumor into the circulation system, and may then combine with the vascular wall of the organ that has high expression levels of CXCL12 (19,8). By this mechanism, the migrating cells could continue to proliferate in the new organ and subsequently metastasize to another location.

CXCR4 levels have been detected as overexpressed in pancreatic cancer tissues and exhibit a close association with the differentiation, metastasis, growth and prognosis of pancreatic cancer $(8,9,20,21)$. The results of the present study demonstrated that the expression levels of CXCR4 in tumor tissues were increased compared with in normal tissues, indicating that CXCR4 may be involved in the development of pancreatic cancer. In addition, the overexpression of CXCR4 in metastatic lymph nodes and distant metastasis was also associated with the TNM stage. Therefore, the overexpression of CXCR4 could contribute to the early diagnosis of patients with pancreatic cancer. The expression of CXCR4 
Table I. CXCR4/CXCL12 expression in various tissues.

\begin{tabular}{|c|c|c|c|c|}
\hline \multirow[b]{2}{*}{ Tissue } & \multicolumn{2}{|c|}{ CXCR4 expression } & \multicolumn{2}{|c|}{ CXCL12 expression } \\
\hline & Negative & Positive & Negative & Positive \\
\hline Pancreatic tumor & 26 & 34 & 8 & 52 \\
\hline Paracancerous tissue & 30 & 30 & 9 & 51 \\
\hline Lymph node & 28 & 32 & 12 & 48 \\
\hline Surrounding tissue & 49 & 11 & 27 & 33 \\
\hline
\end{tabular}

CXCR, C-X-C motif chemokine receptor.

Table II. Correlation of CXCR4 expression with clinicopathological factors.

\begin{tabular}{|c|c|c|c|c|}
\hline Characteristics & Case & CXCR4 (-) & CXCR4 (+) & P-value \\
\hline Age, years & & & & 0.714 \\
\hline$<60$ & 33 & 15 & 18 & \\
\hline$\geq 60$ & 37 & 11 & 16 & \\
\hline Gender & & & & 0.944 \\
\hline Female & 28 & 12 & 16 & \\
\hline Male & 32 & 14 & 18 & \\
\hline Tumor location & & & & 0.121 \\
\hline Head of pancreas & 41 & 15 & 26 & \\
\hline Others & 19 & 11 & 8 & \\
\hline Differentiation & & & & 0.228 \\
\hline Well/moderate & 33 & 12 & 21 & \\
\hline Poor & 27 & 14 & 13 & \\
\hline Pathological type & & & & $0.037^{\mathrm{a}}$ \\
\hline Adenocarcinoma & 54 & 21 & 33 & \\
\hline Others & 6 & 5 & 1 & \\
\hline Lymph node stage & & & & $0.001^{\mathrm{a}}$ \\
\hline Negative & 25 & 19 & 6 & \\
\hline Positive & 35 & 7 & 28 & \\
\hline TNM stage & & & & $0.031^{\mathrm{a}}$ \\
\hline I+II & 23 & 14 & 9 & \\
\hline III+IV & 37 & 12 & 25 & \\
\hline Tumor size, $\mathrm{cm}$ & & & & 0.111 \\
\hline$\leq 3.0$ & 18 & 5 & 13 & \\
\hline$>3.0$ & 42 & 21 & 21 & \\
\hline
\end{tabular}

CXCR4, C-X-C motif chemokine receptor 4; TNM, tumor node metastasis.

was low in normal tissues (positive rate $=18.3 \%$ ), while it was high in pancreatic cancer tissues $(56.7 \%)$, paracarcinoma tissues $(50.0 \%)$ and peripheral lymph nodes (53.3\%), indicating that the deregulation of CXCR4 may be a potential mechanism by which the growth and migration of cancer is regulated. In addition, high expression levels of CRXC4 was also closely associated with the biological effects of pancreatic cancer (pathological type, lymph node stage, and TNM stage; Table II).
There are currently few studies investigating CXCL12 and tumor lesion, and studies have identified that there was abnormal expression of CXCL12 in certain types of cancer, including ovarian and pancreatic cancer and glioma (22-24). Several studies have demonstrated that increased expression levels of CXCL12 in the bone marrow could inhibit tumor cell migration $(23,25)$, and that the progression of high-grade glioma with high expression of CXCL12 would be more rapid than normal (24). In a previous study investigating 
Table III. Analysis of CXCR4 expression levels with biomarkers using the Spearman's test.

\begin{tabular}{lcc}
\hline & \multicolumn{2}{c}{ CXCR4 expression } \\
\cline { 2 - 3 } Biomarker & r-value & P-value \\
\hline VEGF-C & 0.417 & $0.001^{\mathrm{a}}$ \\
$\beta$-catenin & 0.368 & $0.004^{\mathrm{a}}$ \\
Ki-67 & 0.316 & $0.014^{\mathrm{a}}$ \\
MMP-2 & 0.284 & $0.028^{\mathrm{a}}$ \\
\hline
\end{tabular}

CXCR4, C-X-C motif chemokine receptor 4; VEGF-C, vascular endothelial growth factor-C; MMP-2, matrix metalloproteinase-2.

CXCL12/CXCR4, the origin of CXCL12 was suggested to be associated with mesenchymal cells, as a result of the paracrine mechanism of CXCL12, and mesenchymal cells may be involved in tumor cell invasion through the CXCR4/CXCL12 axis (25). Another study hypothesized that CXCL12 may restrain the tumor immune response by blocking the maturation of dendritic cells and by prompting tumor proliferation (26). Therefore, the present study investigated the correlation between the expression of CXCL12 and lymph node metastasis, and subsequently demonstrated this association. In addition, the present study also identified that the level of CXCL12 expression in the tissues surrounding the tumor was increased compared with in normal and noncancerous tissues. These results indicated that CXCL12 performs a vital role in tumor progression, particularly in the process of lymph node metastasis.

A previous study on angiogenesis identified that VEGF was able to promote endothelial cells (EC) to express CXCR4 (27), and a subsequent study confirmed that CXCL12 could induce ECs to express VEGF $(6,28)$. It may therefore be considered that CXCL12/CXCR4 and VEGF form a feedback loop of paracrine signaling in order to coordinate the procedure of angiogenesis and to enhance their biological function. In the present study, VEGF-C exhibited higher levels of expression in tumor-associated tissues compared with in noncancerous pancreatic tissue, which was correlated with the expression levels of CXCR4 $(r=0.556 ; \mathrm{P}<0.001)$. This result indicates that VEGF-C and CXCR4 collaboratively prompt the invasion and metastasis of pancreatic cancer. In addition, the present study also investigated the positive correlation between $\mathrm{Ki}-67$ and CXCR4 ( $r=0.316 ; \mathrm{P}=0.014$ ), and suggested that $\mathrm{CXCR} 4$ may be able to facilitate tumor cell growth. It is generally considered that the binding of CXCL12 and CXCR4 could elevate MMP-2 expression and inhibit the secretion of tissue inhibitors of MMPs (29).

In addition, it has been revealed that the combination of CXCL12 and CXCR4 can activate nuclear factor- $\mathrm{\kappa B}(\mathrm{NF}-\mathrm{\kappa B})$, which significantly increases the secretion of MMP-2 (30), a result concordant with those of the present study. The levels of CXCR4 and MMP-2 protein expression are correlated in pancreatic cancer $(\mathrm{r}=0.284 ; \mathrm{P}=0.028)$, and each were observed to be capable of promoting tumor cell invasion and metastasis and could be utilized as a criteria for evaluating the biological
Table IV. Analysis of CXCR4 with biomarkers by logistic regression analysis.

\begin{tabular}{lccc}
\hline Biomarker & Coefficient $(\beta)$ & P-value & OR \\
\hline VEGF-C & 1.722 & $0.005^{\mathrm{a}}$ & 5.594 \\
$\beta$-catenin & 1.086 & 0.275 & 2.961 \\
Ki-67 & 1.196 & $0.047^{\mathrm{a}}$ & 3.307 \\
MMP-2 & 0.438 & 0.498 & 1.549 \\
\hline
\end{tabular}

${ }^{\mathrm{a}} \mathrm{P}<0.05$. CXCR4, C-X-C motif chemokine receptor 4; VEGF-C, vascular endothelial growth factor-C; MMP-2, matrix metalloproteinase-2; OR, odds ratio.

behavior of pancreatic tumors. Results of in vitro experiments in pancreatic cancer indicated that if CXCR4 was not expressed, the activity of Wnt was suppressed; therefore, the target gene of Wnt//-catenin would be downregulated, ultimately preventing tumor invasion or migration (31). Levels of CXCR4 expression were determined to be significantly correlated with the expression levels of $\beta$-catenin $(r=0.292$; $\mathrm{P}=0.024)$. Therefore, CXCL12/CXCR4 may be able to regulate the $\mathrm{Wnt} / \beta$-catenin signal transduction pathway in order to alter the performance of corresponding factors. In addition, the correlation of $\beta$-catenin expression with MMP-2 was observed to be significant $(r=0.308 ; P=0.017)$, which indicates that $\beta$-catenin may upregulate members of the MMP family's expression to enhance the decomposition of ECM and vascular basement membrane in order to provoke the invasion and metastasis of pancreatic tumors.

The association between the CXCR4/CXCL12 axis and the biological behavior of pancreatic tumors has been established. All pancreatic tumor tissues express CXCR4 and CXCL12 at higher expression levels compared with those in noncancerous tissues. The CXCL12/CXCR4 axis is involved in the angiogenesis of pancreatic cancer tumors, promotes tumor cell migration and cellular proliferation and inhibits apoptosis. It is now believed that VEGF-C, Ki-67 and CXCR4 can be considered indicators of tumor therapeutic efficacy and can determine whether tumor metastasis occurs. The aforementioned biological effects of the CXCR4/CXCL12 axis may be inhibited by VEGF-C antagonists, and may then become a novel target in the directed therapy of patients with pancreatic cancer.

The CXCL12/CXCR4 axis performs an essential role in the behavior of pancreatic cancer. VEGF-C and Ki-67 are two important biomarkers, through which CXCR4 may induce the metastatic behavior of pancreatic cancer cells. Therefore, inhibitors of angiogenesis continue to be effective agents for treating pancreatic cancer.

\section{References}

1. Siegel RL, Miller KD and Jemal A: Cancer statistics, 2016. CA Cancer J Clin 66: 7-30, 2016.

2. Li D, Xie K, Wolff R and Abbruzzese JL: Pancreatic cancer. Lancet 363: 1049-1057, 2004.

3. Kim VM and Ahuja N: Early detection of pancreatic cancer. Chin J Cancer Res 27: 321-331, 2015. 
4. Sheffield KM, Crowell KT, Lin YL, Djukom C, Goodwin JS and Riall TS: Surveillance of pancreatic cancer patients after surgical resection. Ann Surg Oncol 19: 1670-1677, 2012.

5. Vandercappellen J, Van Damme J and Struyf S: The role of CXC chemokines and their receptors in cancer. Cancer Lett 267: 226-244, 2008

6. Chatterjee S, Behnam Azad B and Nimmagadda S: The intricate role of CXCR4 in cancer. Adv Cancer Res 124: 31-82, 2014.

7. Zhao H, Guo L, Zhao H, Zhao J, Weng H and Zhao B: CXCR4 over-expression and survival in cancer: A system review and meta-analysis. Oncotarget 6: 5022-5040, 2015.

8. Wu PF, Lu ZP, Cai BB, Tian L, Zou C, Jiang KR and Miao Y: Role of CXCL12/CXCR4 signaling axis in pancreatic cancer. Chin Med J (Engl) 126: 3371-3374, 2013.

9. Cui K, Zhao W, Wang C, Wang A, Zhang B, Zhou W, Yu J, Sun Z and Li S: The CXCR4-CXCL12 pathway facilitates the progression of pancreatic cancer Via induction of angiogenesis and lymphangiogenesis. J Surg Res 171: 143-150, .2011.

10. Bilimoria KY, Bentrem DJ, Merkow RP, Tomlinson JS, Stewart AK, Ko CY and Talamonti MS: Application of the pancreatic adenocarcinoma staging system to pancreatic neuroendocrine tumors. J Am Coll Surg 205: 558-563, 2007.

11. Vincent A, Herman J, Schulick R, Hruban RH and Goggins M: Pancreatic cancer. Lancet 378: 607-620, 2011.

12. Siegel R, Naishadham D and Jemal A: Cancer statistics, 2012. CA Cancer J Clin 62: 10-29, 2012.

13. Stathis A and Moore MJ: Advanced pancreatic carcinoma: Current treatment and future challenges. Nat Rev Clin Oncol 7: $163-372,2010$

14. Sharma C, Eltawil KM, Renfrew PD, Walsh MJ and Molinari M: Advances in diagnosis, treatment and palliation of pancreatic carcinoma: 1990-2010. World J Gastroenterol 17: 867-897, 2011.

15. Jiang YM, Li G, Sun BC, Zhao XL and Zhou ZK: Study on the relationship between cxcr4 expression and perineural invasion in pancreatic cancer. Asian Pac J Cancer Prev 15: 4893-4896, 2014

16. Xu C, Zhao H, Chen $\mathrm{H}$ and Yao Q: CXCR4 in breast cancer: Oncogenic role and therapeutic targeting. Drug Des Devel Ther 9: 4953-4964, 2015

17. Tsai MF, Chang TH, Wu SG, Yang HY, Hsu YC, Yang PC and Shih JY: EGFR-L858R mutant enhances lung adenocarcinoma cell invasive ability and promotes malignant pleural effusion formation through activation of the CXCL12-CXCR4 pathway. Sci Rep 5: 13574, 2015

18. Deng L, Shang Y, Guo S, Liu C, Zhou L, Sun Y, Nie Y, Fan D, Lu Y and Guo X: Ran GTPase protein promotes metastasis and invasion in pancreatic cancer by deregulating the expression of AR and CXCR4. Cancer Biol Ther 15: 1087-1093, 2014.

19. Sun X, Cheng G, Hao M, Zheng J,Zhou X, Zhang J, Taichman RS, Pienta KJ and Wang J: CXCL12/CXCR4/CXCR7 chemokine axis and cancer progression. Cancer Metastasis Rev 29: 709-722, 2010 .
20. Wehler T, Wolfert F, Schimanski CC, Gockel I, Herr W, Biesterfeld S, Seifert JK, Adwan H, Berger MR, Junginger T, et al: Strong expression of chemokine receptor CXCR4 by pancreatic cancer correlates with advanced disease. Oncol Rep 16 1159-1164, 2006.

21. Krieg A, Riemer JC, Telan LA, Gabbert HE and Knoefel WT: CXCR4-A prognostic and clinicopathological biomarker for pancreatic ductal adenocarcinoma: A Meta-Analysis. PLoS One 10: e0130192, 2015.

22. Zhou Y, Larsen PH, Hao C and Yong VW: CXCR4 is a major chemokine receptor on glioma cells and mediates their survival. J Brol Chem 277: 49481-49487, 2002.

23. Lapidot $\mathrm{T}$ and Kollet O: The essential roles of the chemokine SDF-1 and its receptor CXCR4 in human stem cell homing and repopulation of tranaplanted immune-deficient NOD/SCID and NOD/SCID/B2m(null) mice. Leukemia 16: 1992-2003, 2002.

24. Salmaggi A, Gelati M, Pollo B, Frigerio S, Eoli M, Silvani A, Broggi G, Ciusani E, Croci D, Boiardi A and De Rossi M: CXCL12 in malignant glial tumors: A possible role in angiogenesis and cross-talk between endothelial and tumoral cells. J Neurooncol 67: 305-317, 2004.

25. Zhong W, Chen W, Zhang D, Sun J, Li Y, Zhang J, Gao Y, Zhou W and Li S: CXCL12/CXCR4 axis plays pivotal roles in the organ-specific metastasis of pancreatic adenocarcinoma: A clinical study. Exp Ther Med 4: 363-369, 2012.

26. Zou W, Machelon V, Coulomb-L'Hermin A, Borvak J, Nome F, Isaeva T, Wei S, Krzysiek R, Durand-Gasselin I, Gordon A, et al: Stromal-derived factor- 1 in human tumors recruits and alters the function of plasmacytoid precursor dendritic cells. Nat Med 7: 1339-1346, 2001.

27. Salvucci O, Yao L, Villalba S, Sajewicz A, Pittaluga S and Tosato G: Regulation of endothelial cell branching morphogenesis by endogenous chemokine stromal-derived factor-1. Blood 99: 2703-2711, 2002

28. Wang Z, Ma Q, Li P, Sha H, Li X and Xu J: Aberrant expression of CXCR4 and $\beta$-catenin in pancreatic cancer. Anticancer Res 33: 4103-4110, 2013.

29. Lane WJ, Dias S, Hattori K, Heissig B, Choy M, Rabbany SY, Wood J, Moore MA and Rafii S: Stromal-derived factor 1-induced megakaryocyte migration and platelet production is dependent on matrix metalloproteinases. Blood 96: 4152-4159, 2000.

30. Yuecheng Y and Xiaoyan X: Stromal-cell derived factor-1 regulates epithelial ovarian cancer cell invasion by activating matrix metalloproteinase-9 and matrix metalloproteinase-2. Eur J Cancer Prev 16: 430-435, 2007.

31. Wang Z, Ma Q, Liu Q, Yu H, Zhao L, Shen S and Yao J: Blockade of SDF-1/CXCR4 signalling inhibits pancreatic cancer progression in vitro via inactivation of canonical Wnt pathway. Br J Cancer 99: 1695-1703, 2008. 